Techniques \& Culture

\title{
Les dispositifs du Net art
}

Entre configuration technique et cadrage social de l'interaction

Jean-Paul Fourmentraux

\section{(2) OpenEdition}

Journals

Édition électronique

URL : https://journals.openedition.org/tc/2872

DOI : $10.4000 /$ tc. 2872

ISSN : 1952-420X

Éditeur

Éditions de l'EHESS

Édition imprimée

Date de publication : 12 décembre 2007

Pagination : 269-302

ISSN : 0248-6016

Référence électronique

Jean-Paul Fourmentraux, «Les dispositifs du Net art », Techniques \& Culture [En ligne], 48-49| 2007, mis en ligne le 20 juin 2010, consulté le 29 septembre 2022. URL : http://journals.openedition.org/tc/ 2872 ; DOI : https://doi.org/10.4000/tc. 2872 


\title{
Jean-Paul Fourmentraux*
}

\author{
Les dispositifs du Net art \\ Entre configuration technique \\ et cadrage social de l'interaction
}

\begin{abstract}
La pratique du Net art radicalise la question du potentiel communicationnel d'un média Internet - qui constitue tout à la fois le support technique, l'outil créatif et le dispositif social de l'ouvre. Les technologies de linformation et de la communication (TIC) placent en effet l'ouvre d'art au coeur d’une négociation socialement distribuée entre lartiste et le public. Larticle est focalisé sur cette construction collective du Net art et sur ses mises en scènes. II montre le travail artistique et les configurations techniques qui composent les cadres de l'expérience du Net art. Une typologie de ces dispositifs offre de distinguer des figures de linteractivité, telles qu'elles sont prévues au cœur de l'environnement technique, et les modes dinteraction qu'elles déploient en direction du public.
\end{abstract}

Coordination, dispositifs sociotechniques, Internet, médiation, travail artistique, sociologie pragmatiste

Depuis le milieu des années 1990 , le Net art distingue les créations interactives conçues par, pour et avec le réseau Internet, vis-à-vis des formes d'art plus traditionnelles simplement transférées sur des sites-galeries et autres musées virtuels ${ }^{1}$. Toutefois, si le Net art tend désormais à désigner des productions possédant un minimum de caractéristiques communes, cette appellation générique ne doit pas donner lillusion d’une unité des pratiques. Dans ce contexte, naissent des projets multiformes - environnements navigables, programmes exécutables, formes altérables- qui renouvellent

\footnotetext{
* GERIICO Université de Lille 3, domaine universitaire du Pont de Bois, Rue du Barreau, BP 60149, 59653 Villeneuve d'Ascq. Courriel : jean-paul.fourmentraux@univ-lille3.fr.

${ }^{1}$ Au terme de dix années d'existence (1995-2005), le vocable Net art s'est très largement imposé en Europe et en France au détriment d'autres qualifications comme « art Internet », « art réseau », « cyberart », «web art », qui manquaient à clairement distinguer l'art sur le réseau de l'art en réseau.
} 
pour bonne part les modes d'évaluation et d'appréhension de l'œuvre d'art. Pour rendre compte de cette diversité, une typologie peut mettre l'accent sur des aspects différents de l'œuvre. Sa forme - sa structure ou ses composantes plastiques - s'accommode tout à fait de l'évaluation stylistique qualitative. Cependant, afin de privilégier l'examen des usages, nous allons choisir ici d'éviter tout critère esthétique, l'objectif étant de comprendre comment s'organisent et se déploient les moyens mis en œuvre pour concevoir et expérimenter les œuvres du Net art. Mais là encore, diverses modalités de catégorisation sont possibles. Partant de ce qui est donné à voir à l'écran ${ }^{2}$, une classification peut être fondée sur la marge de participation que les œuvres prévoient. Cette classification a l'avantage de pointer les différences les plus significatives entre les œuvres et les types correspondants de participation du visiteur. Mais son défaut essentiel est d'en rester à la partie émergée, autrement dit à ce qui est visible à l'écran. Or, l'examen approfondi des sites d'artistes montre que, dans certains cas, l'œuvre se trouve non plus seulement dans ce qui est donné à voir mais aussi dans le dispositif qui la fait exister. La visibilité sur l'écran n'étant que la face apparente de toute une infrastructure technique et informationnelle. Pour cette raison, à une catégorisation fondée sur ce que l'on attend du participant et/ou sur la forme de l'œuvre, il faut préférer celle fondée sur la manière dont est agencé le site. C'est sur cette seconde option que repose l'approche proposée ici. Fonder cette typologie sur la manière dont est agencé le site permet, en outre, d'éviter de repérer immédiatement - et donc de définir a priori- ce qu'est et où est l'œuvre. Dans ce contexte, l'emploi du concept de dispositif reprend et prolonge les figures artistiques de linstallation et de la performance: il permet de montrer successivement la configuration et le fonctionnement de l'œuvre, ainsi que la distribution des rôles et les différentes formes d'énonciation qu'il installe symétriquement pour faire œuvre. Cette question du dispositif est devenue centrale depuis de nombreuses années en esthétique, en critique et en histoire de l'art, depuis l'étude classique d'Umberto Eco, L'Euvre ouverte (1965), qui montrait l'importance historique et théorique de la question de la participation du spectateur à l'œuvre. On la trouve ensuite chez Louis Marin (I994), chez Daniel Arasse (1999), bien avant sont entrée dans le cadre de la théorie de L'Esthétique

\footnotetext{
2 C'est l'orientation empruntée par les recherches sur «l'écrit d'écran », initiées par Jean-Yves Jeanneret (2000) et Emannuel Souchier (2001).
} 
relationnelle de Nicolas Bourriaud (1998) ou de l'agency d'Alfred Gell (1998). Symétriquement, depuis l'étude liminaire de Barthes (1975) qui a proposé de concevoir le dispositif cinématographique au travers de sa réception négociée avec le public, de nouvelles recherches centrées sur l'impact et les usages des technologies de linformation et de la communication ont enrichi linterprétation pragmatique de ce concept de dispositif : voir Jacquinnot et Monnoyer (I999), Duguet (I988, 2002), Boissier (2004), Fourmentraux (2005). Cette acception du dispositif voisine enfin au cœur du présent texte d'autres concepts sociologiques, avec lesquels elle conjugue une vision du social en acte et une conception délibérément pragmatique de la sociologie : en empruntant aux concepts d'attachement (Latour 1999), de configuration ou d'agencement (Strauss 1992) et de mise en œuvre (Goodman I996; Genette 1996), l'objectif de servir une appréhension appariée de l'écriture et de l'action en art. L'analyse visant à éclairer l'action de ceux qui conçoivent, là où se joue la médiation, non pas avec, mais, dans l'environnement technique (Norman I993; Hennion, I993; Latour 1994 ; Thévenot 1990, 1994).

En résumé, le dispositif supporte simultanément linteractivité avec la machine et linteraction auteur(s)/acteur(s) que déploient ses usages. Cet article adopte le parti pris analytique d'isoler chacun de ces mouvements pour mieux en saisir la spécificité et les modes d'action. Il propose de sérier les productions du Net art en distinguant les «figures de linteractivité machinique » et les « modes d'interaction » avec le public, mis en œuvre dans trois types principaux de dispositifs : l'œuvre de contamination médiatique, l'œuvre de programmation algorithmique et l'œuvre de communication interactive. Il en résulte trois engagements "esthétiques", focalisés sur le code informatique, le programme et linteractivité 3 .

\section{L'œuvre médiatique}

Linvestigation médiatique marque les premières réalisations liées à une innovation technique. Une même détermination touche, au début de leur histoire, les médiums photographique, cinématographique et

\footnotetext{
3 Le matériel empirique sur lequel s'appuie ce texte est issu d'une enquête effectuée dans le cadre plus général d'une thèse de sociologie. Les cas présentés ici sont choisis parmi un corpus de 50 dispositifs Net art qui a servi une typologie des figures de l'interactivité et des modes d'interaction entre l'artiste, l'œurre et son public. L'analyse a été complétée par des entretiens avec leurs auteurs. Pour une présentation détaillée de ce travail, voir Jean-Paul Fourmentraux (2005).
} 
vidéographique, tour à tour explorés, contournés et détournés par la pratique artistique expérimentale ${ }^{4}$. Les Net artistes vont d'abord dénoncer la prégnance d'un langage quasi exclusif 's'organisation des données hypertextuelles qui contribue, selon eux, à accentuer le caractère uniforme de la majorité des sites Web, dans leur agencement aussi bien que dans l'apparence de leurs interfaces. L'approche artistique consistera à contourner ces prescriptions d'emplois (Akrich 1990, I993; Woolgar I99I; Thévenot 1990, 1994) visant à discipliner les usages et parcours au sein des sites Web : les liens soulignés en bleu, les images cliquables, les zones title et body. Répondant à cette normalisation de la navigation, la plupart des sites Web ont en effet été développés en exploitant au minimum et de manière essentiellement fonctionnelle les potentialités du médium. Malgré l'importation de nombreux plug-ins (modules logiciels annexes) permettant l'enchâssement d'éléments multimédias tels que le son (Real audio) et la vidéo (Quicktime), de nombreux sites ont formellement reproduit des schémas visuels connus et empruntés à d'autres supports pour la présentation des pages et des textes dans un design proche de celui du livre ou du magazine sur papier. Les Net artistes vont donc proposer des voies alternatives à ces dérives fonctionnelles, telles que le "pointer-cliquer" comme convention de navigation, la distribution rigide de l'information, sa réception passive des informations, etc.

Les dispositifs de piratage

Un nombre important d'artistes du réseau revendiquent dans le monde de l'art une implication parasitaire ${ }^{6}$. L'entité artistique Jodi dispose sur le réseau

\footnotetext{
${ }^{4}$ Les premières pièces vidéo de Nam June Paik ou celles de Wolf Vostell se sont notamment attachées à détruire la télévision, physiquement (sculptures vidéo) autant que symboliquement, en intervenant à même la matière du médium par des altérations du signal vidéo. La télévision, le meuble lui-même, l'écran, le tube cathodique, le signal vidéo et son indéfinition, sa fébrilité et sa luminance étaient pris à la fois comme l'objet et le matériau de l'investigation artistique.

5 Il s'agit du Hypertext Markup Language (HTML). Même si aujourd'hui de nouveaux langages d'implémentations de l'hypertexte sont utilisés comme le $\mathrm{C}++$ ou le Java, eux-mêmes parfaitement compatibles avec le Web mais qui exigent un niveau de compétences incomparablement plus élevé en programmation informatique.

${ }^{6}$ Pour un premier manifeste du Net art «activiste» voir Joachim Blank : http://www.irational.org/cern/Netart.txt.
} 
des travaux ${ }^{7}$ qui empruntent leur réalité et leur forme aux actions et comportements déviants des pirates de l'informatique: les hackers. Cette démarche d'emprunt a pour objet l'incident, le bug et l'inconfort technologique. Leurs dispositifs agissent sur la structure du langage HTML (cf. note 5) par altération du code et transformation des balises qui permettent aux internautes la reconnaissance et l'agencement des différents éléments graphiques : tant au niveau de la mise en page que de l'intégration des composantes multimédias, du son, de l'image, de la vidéo ${ }^{8}$. L'information y est partielle, non visible. Le parcours en est chaotique, sans cohérence a priori et conduit à dériver d'un lien à un autre, sans que l'on puisse percevoir la structure du dispositif. Des interfaces de brouillage confrontent le visiteur à l'apparition constante de messages d'alerte, associés à la perte de contrôle de l'ordinateur qui ne répond plus à aucune commande.

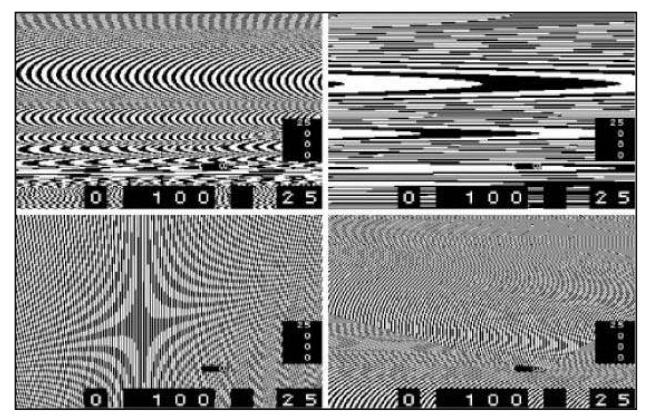

Figure 1. Jodi, OSS,

http://www.oss.Jodi.org0

Le site http://oss.Jodi.org confronte le visiteur à la prolifération de petites fenêtres de navigation qui successivement s'ouvrent, se referment et défilent de manière aléatoire à un rythme frénétique sur toute la surface de l'écran. Il est impossible de les maîtriser. À mesure que l'on s'attache à les

${ }^{7}$ Cf. Jodi, Portail, http://www.Jodi.org; Jodi, OSS, http://www.oss.Jodi.org; Cf. Jodi, Error 404, http://www.404.Jodi.org

8 Pour ces artistes, un écran qui tremble est un matériau. Les fonctions informatiques Undo, Select, Delete, ou Send ont de réelles propriétés avec de réels effets et conséquences. L'erreur système 404 qu'ils affichent en point d'entrée de leur site devient un leitmotiv créatif. Leurs projets intègrent en effet dans leur développement les contraintes du médium : l'exigence de fluidité impose des fichiers très légers et engage les artistes dans une voie à contre-courant de l'évolution technologique. 
fermer une à une, d'autres surviennent dans un nombre toujours plus important. L'identification et la neutralisation de la fenêtre principale, le fait de mettre une autre adresse ou de solliciter un autre lien n'y changent rien, des fenêtres auxiliaires continuent d'envahir l'écran, rendant difficile la lecture et la navigation. L’ultime solution reste done de quitter le navigateur. Pour cela, seul le raccourci clavier qui permet de "forcer à quitter" l'application" est efficace, les autres touches du clavier étant rendues inopérantes par le dispositif.

Un second processus accompagne le dispositif Oss. Le programme développé par Jodi semble ordonner au navigateur d'accomplir une tâche spécifique sans que linternaute en ait décidé et sans même qu'il s'en rende compte. En effet, au fil du temps que nécessite la navigation dans le site et la tentative de se sortir du piège tendu par Oss I, le dispositif programmé par Jodi invite le navigateur à télécharger des fichiers-applications qui viennent se loger dans le disque dur ou sur le bureau de l'ordinateur-client. Mais cette opération peut tout à fait demeurer invisible et passer inaperçue. La machine qui paraît naturellement en activité, mobilisée par l'affichage des interfaces de brouillage d'Oss I, ne dévoile aucunement au visiteur de l'œuvre ce qui se trame en tâche de fond, dans les coulisses du dispositif. La fenêtre du gestionnaire de téléchargement n'apparaissant pas par défaut à l'écran, l'opération peut en effet être ainsi dissimulée. Dans le meilleur des cas, ce n'est donc qu'après avoir quitté le navigateur que l'internaute peut apercevoir les icones d'applications téléchargés sur son bureau informatique. Et parfois, ce n'est que bien plus tard quil pourra les découvrir, par hasard, dans le disque dur de son ordinateur. Lorsque l'internaute se risque à commander l'ouverture d'un de ces icones, l'application simule de façon violente et inattendue le crash de l'ordinateur. Dans le même temps, elle neutralise toutes les commandes habituelles, la mobilité de la souris ainsi que l'action des touches du clavier. Cette expérience met ainsi l'accent sur la paranoïa qui peut entourer la relation de l'internaute à son ordinateur : la peur des virus, la crainte du bug qui conduit la machine à planter en entraînant parfois la perte irrémédiable des données, jusqu'au crash du disque dur. Jodi amplifie et accentue cette tension en adoptant un

\footnotetext{
9 À titre d'exemple, cette fonction pour un ordinateur Macintosh demande d'enfoncer simultanément les touches pomme+alt+esc.
} 
comportement proche de celui des hackers, axé sur l'intrusion et la contamination $^{10}$.

Linterface des navigateurs constitue un autre objet d'expérimentation. Certains projets prennent pour cible les technologies de repérage et d'accès à l'information sur Internet. Certains projets initient des destructurations de l'information. C'est le cas, par exemple, des navigateurs subversifs développés par lartiste Mark Napier. Son Shredder ${ }^{11}$ part du constat critique que l'affichage sur le Web repose sur une illusion de solidité et de permanence. Lorsqu'on est amené à développer un site, on se rend vite compte, en effet, de la fragilité de ses composantes et de leur agencement. D'un navigateur à l'autre, et selon la résolution de l'écran, l'affichage des pages et des liens peut être altéré. Derrière lilllusion graphique, le Web soustend une succession de fichiers-textes, contenant le code HTML, lequel transmet aux navigateurs des instructions de mise en pages, d'intégration et de disposition des différents éléments multimédias. Les pages Web représentent en ce sens la partie visible du code HTML après que le logiciel de navigation en a interprété les instructions ${ }^{12}$. De ce point de vue, les browsers apparaissent comme autant d'organes de perception au travers desquels nous voyons le Web. Ils filtrent et organisent les informations présentes sur un nombre exponentiel d'ordinateurs dispersés dans le monde. Le projet de Mark Napier propose, dans ce contexte, un moteur de recherche subversif qui vient altérer le code HTML avant même que le logiciel de navigation ne puisse le lire. Le Shredder s'immisce dans l'interstice entre le code écrit par le concepteur d'un site Web et son affichage ou sa traduction graphique par le navigateur. Ce dispositif souligne l'équilibre instable de la traduction informatique et conduit le visiteur à percevoir le système de représentation du Web, en l'affranchissant des mécanismes obligatoires de la navigation passive.

Au-delà de cette réflexion sur lagencement des données et leur affichage médié par le navigateur, d'autres dispositifs visent la distorsion du contenu de linformation. C'est par exemple le cas des ready made de l'artiste slovène

\footnotetext{
${ }^{10}$ Les concepteurs de Jodi pensent en effet qu'ils parviennent à être très proches des gens et de leur public lorsqu'ils s'immiscent dans leur bureau (au sens informatique du terme) : «Quand les regardeurs visitent notre travail, nous sommes à l'intérieur de leur ordinateur ».

${ }^{11}$ Cf. Mark Napier, Shredder, http://potatoland.org/shredder/welcome.html.

12 Cf. Maciej Wisniewski, Netomat, http://www.Netomat.Net/, I/O/D (Mac, U.K. 1997), I/O/D 4: The Web Stalker, http://www.backspace.org/iod/.
} 
Vuk Cosic qui détourne des sites Web institutionnels. On lui doit notamment le piratage du site de la Documenta $X$ de Kassel ${ }^{13}$. Dans une perspective comparable, l'activiste anglais Heath Bunting ${ }^{14}$ et les Américains RTMARK ${ }^{15}$ déploient sur le réseau une logique virale, visant la perversion des communications médiatiques de grandes puissances financières. Enfin, le collectif européen ETOY ${ }^{16}$ a également mené de nombreuses actions en ce sens, au cœur de la bataille politique et économique des noms de domaines sur Internet (DNS, Dot.com) - ces derniers se revendiquant auteurs d'une guerre informationnelle sur le terrain de l'e-business et des nouvelles valeurs financières comme le NASDAQ, etc.

\section{Une esthétique du code informatique}

Bien plus qu'à une esthétique de l'interactivité, ces différents dispositifs confrontent les visiteurs à une esthétique de l'informatique, du code, et parfois du crash. Le code, le programme, le langage HTML constituent la matière numérique prise à la fois comme le matériau et la forme de l'expression plastique ${ }^{17}$. Il s'agit en quelque sorte d'un art de la confrontation médiatique à l'Internet en tant qu'interface technologique, à l'Internet comme média. L'objectif visé est ainsi le détournement des perceptions et des usages familiers de l'écran d'ordinateur, en imposant au public de déconstruire et reconstruire cette surface par lintroduction de différents bruits - même si le plus souvent ici, linternaute demeure extérieur à l'œuvre. Il est la victime et la cible du dispositif et l'accent est porté sur le déterminisme technologique de cette relation. Par conséquent, ces dispositifs engagent une figure de l'interactivité basée sur l'action et la réaction face à l'ordinateur dans des situations limites. L'expérience de ces objets génère chez l'internaute des états d'inconfort technique, amplifie la fragilité de sa relation à la machine (susceptible de boguer à tout moment) et fait de lui une victime du " complot " artistique.

\footnotetext{
${ }^{13}$ Cf. Vuk Cosic, http://www.ljudmila.org/ vuk/ et http://www.ljudmila.org/ vuk/dx/.

${ }^{14} \mathrm{Cf}$. Heath Bunting, http://www.irational.org.

${ }^{15}$ Cf. RTMARK, http://www.rtmark.com.

${ }^{16} \mathrm{Cf}$. Etoy, http://www.etoy.com.

17 Pour d'autres expérimentations basées sur l'esthétique de l'interface, voir également les dispositifs de l'artiste russe Alexeï Shulgin, http://www.easylife.org, de l'artiste français D2B, http://www.d2b.org, et des Allemands Joachim Blank et Karl Heinz Jeron, http://sero.org/dyt/.
} 


\section{L'œuvre algorithmique}

En poursuivant cette expérimentation focalisée sur le média, l'art algorithmique promeut une remontée du code au programme et déplace le pôle d'investigation de linterface affichée vers le moteur de l'application. Certains artistes proposent en effet d'investir le domaine circonscrit des applications logicielles et environnements algorithmiques. L'Art Bit Collection $^{18}$ de l'International Computer Consortium de Tokyo (ICG) regroupe des travaux qui explorent en cette direction les recherches du Net art. Les thématiques présentées proposent un parcours dans la création numérique couvrant l'ensemble de la deuxième moitié des années 1990. Elles rassemblent principalement des expérimentations autour des langages de programmation, des environnements logiciels (Network community), des applicatifs de visualisation des coulisses du World Wide Web et, enfin, des applications détournées de logiciels interactifs.

Les dispositifs de programmation

- Les environnements visuels de programmation

À linverse des méthodes et des conventions de programmation basées sur le langage informatique, ces travaux offrent un environnement de programmation centré autour du visuel. Sans connaissance nécessaire des langages de programmation, les utilisateurs peuvent, par action et manipulation d'objets sur l'écran, programmer l'ordinateur et lui faire exécuter différentes tâches ${ }^{19}$.

- Les environnements de média-programmation

\footnotetext{
18 "In the art world, a work of art is called an "art piece". The word "piece" designates a thing that actually exists, but since software creations exist only as binary data, calling them an "art piece" seems wrong. Substituting "bit" for "piece", we have decided to call such a work an "art bit" ». Manifeste de l'exposition "Art.bit collection », June 21 - August 11, 2002 @ ICC.

${ }^{19}$ Cf. Alexander Repenning (Mac, U.S.A. 1989), AgentSheets, http://www.agentsheets.com/, Ken Kahn (Win, U.S.A.) ToonTalk, http://www.toontalk.com/, Kakuya Yamamoto (Win, Japan), 3D-Visulan, http://ryujin.kuis.kyoto-u.ac.jp/ylab/yamakaku/Visulan/, Kuniaki Watanabe (PlayStation, Japan 1999), PaneKit, http://www5d.biglobe.ne.jp/ owhari/panekit/panekit_index.html, CogniToy (Win, U.S.A. 2000), MindRover, http://www.mindrover.com/, Yumiko Tanaka (Java, Win, Japan 2000), drawing blocks, http://mutoh.imrf.or.jp/tanaka/
} 
Il s'agit là d'environnements logiciels permettant aux utilisateurs linsertion, la création et la restructuration de sons et dimages ${ }^{20}$.

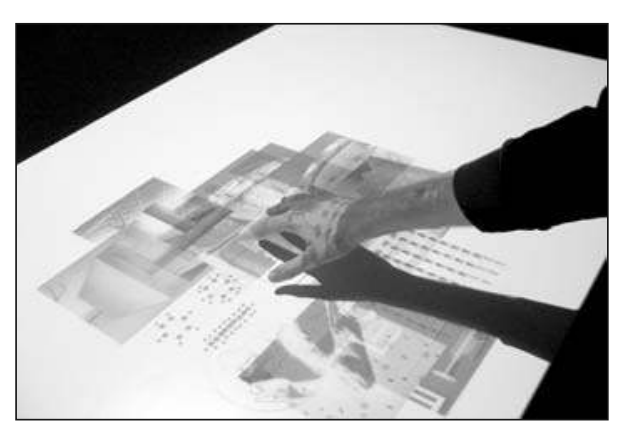

Figure 2. Douglas Stanley, Concrescence, www.abstractmachine.net

- Les applicatifs de connexion inter-uFitilisateurs (CommunityWare) Ces logiciels de communication assistent les utilisateurs dans la formation de communautés interactives. La présentation et l'expérimentation de ces programmes donnent lieu à des workshops collectifs au cours desquels les communautés se constituent ${ }^{21}$.

- Les environnements virtuels

Les utilisateurs visitent des environnements imaginaires disposés dans l'ordinateur, ils créent des créatures qui pourront habiter ces espaces. Ces créatures artificielles deviendront les résidents permanents de ces environnements ${ }^{22}$.

- Les dispositifs de visualisation des dessous du réseau.

\footnotetext{
${ }^{20} \mathrm{Cf}$. Miller Puckette and David Zicarelli (Mac, France), MAX/MSP http://www.cycling74.com/products/max.Html, Stephane Rollandin (France 2000), GeoMaestro, http://www.zogotounga.Net/GM/eGM0.Html Netochka Nezvanova (MAX, Mac, nSk state 1999-2000), nato, http://www.eusocial.com/, Steim (Mac, Netherland), BigEye, http://www.steim.nl/bigeye.html /

${ }^{21}$ Cf. Graham Harwood (Win, U.K.), Linker, http://www.linker.org.uk/

${ }^{22}$ Cf. Antoine Schmitt, Avec détermination, mai 2000, http://www.gratin.org/as/avecdetermination/index.html, Sodaconstructor, sodarace (projector(3screens), table) (Win, U.K.), sodaplay, http://www.sodaplay.com/, Kenichirou Shii (Win, Japan), Sand, http://www.kdn.gr.jp/ shii/sand/index.html/.
} 
Ils matérialisent à l'écran d'ordinateur l'ensemble des informations et données utilisées dans, par et hors du dispositif. L'œuvre résulte ici de ce flot de données hétérogènes rendues visibles ${ }^{23}$.

- Les détournements de logiciels-auteurs

Ils offrent la possibilité de créer, retoucher et agencer des éléments textuels ou graphiques (à linstar des principaux traitements de textes et autres outils de type palette graphique); ces applicatifs proposent une alternative et une perturbation des applications professionnelles ${ }^{24}$.

Ces dispositifs sont done davantage axés sur les applications informatiques à l'usage des internautes, appelées aussi les logiciels auteurs. Le Trace noiser offre par exemple un générateur de clones informationnels qui croise les fonctionnalités du moteur de recherche, les outils statistiques d'indexation et de traçage des réseaux de liens. Selon une stratégie de déstructuration des informations cristallisées sur le Web, l'application explore la toile pour composer un clone identitaire des internautes (databody) à partir des traces numériques, disséminées au fil des liens, qui renseignent sur son activité, ses appartenances, mais également ses parcours sur le Web et ses centres d'intérêts (les traces liées à un nom, e-mails, formulaires de commande, signatures, déclarations d'usage de logiciel, etc. ${ }^{25}$ ). Cette réflexion sur liidentité place la projection de la personnalité dans un entre-deux médiatique qui opère entre une information réelle et une information générée par la machine -une identité fragmentée recomposée à partir du glanage et du réagencement alternatif des sources dispersées retrouvées sur le réseau.

Le Net art promeut de nombreuses applications de ce type, qui simmiscent entre linternaute, les données et la machine. Par exemple, le

\footnotetext{
${ }^{23}$ Cf. Sensorium (Java, Win, Japan, 1996), WebHopper, http://www.sensorium.org/Webhopper/, RSG (Win, U.S.A. 2001), Carnivore, http://www.rhizome.org/carnivore/, Satoru Sugihara (Linux w/NVidia graphic card, Japan 2002), Tokyo Local Webscape, http://www.imrf.or.jp/ sugihara/Webscape/

${ }^{24}$ Cf. Adrian Ward (Mac, U.K. 1999), Autoshop, http://www.signwave.co.uk/products/autoshop/ et (Mac, U.K. 2001) Auto-Illustrator, http://www.auto-illustrator.com/. Voir également, LAN (HTML, Switzerland 2001), TraceNoizer, http://www.tracenoizer.org/ et Kouichirou Eto (Win, Japan 2002), LivingWebBrowser, http://eto.com/2002/LivingWebBrowser/.

${ }^{25}$ Le dispositif use de différents modules logiciels en Open-Source: Puf pour l'extraction de données HTML, Rainbow pour la modélisation statistique, le rapatriement des textes et leur classification, le serveur Apache, les scripts d'exécution Php et Perl, ainsi que Mysql pour la gestion de base de données.
} 
dispositif Carnivore développé par le collectif RSG se veut un outil de surveillance des données en réseaux. Le projet consiste en un applicatif de mise en écoute, depuis un serveur local, de l'ensemble du trafic de l'Internet (e-mails, parcours de navigations, etc.). Le serveur (re)distribue sur le Net, vers un nombre illimité d’interfaces créatives clients, ce flux de données prélevées. Chaque client est configuré pour diagnostiquer, interpréter et animer le trafic de données selon des partis pris divers. Promue lors du dernier festival Ars Electronica, cette installation est un détournement du logiciel DCS Iooo employé par le FBI pour développer l'écoute électronique sur le réseau. Plus récemment, le Net art a intégré et validé dans la sphère artistique des applications étrangère à ses finalités telles que Linux, initié par Linus Torvald (premier prix de l'Ars Electronica, catégorie Net, en I999), ou encore, le WorldWideWeb (1990) de Tim Berners Lee, ainsi que les navigateurs Mosaic (1993) et Netscape (1995) de Marc Andreesen et Eric Bina récompensés sur la scène artistique de l'art algorithmique.

\section{Une esthétique du programme}

L'acte créatif relève dans ce contexte de la conception d'un programme d'ordinateur (un algorithme) dont limplémentation constitue simultanément la source (invisible) et le coeur de l'ouvre plastique. Cette forme d'art algorithmique promeut ainsi une remontée de l'acte créatif en amont de l’ouvre, à ses prémices dans les coulisses de la machine, au travers de l'écriture du programme enfoui et inaccessible. Cette pré-disposition écrite apparente ces projets aux formes antérieures de lart conceptuel, dont la propriété fondamentale réside dans une dialectique de la clôture (écriture) et de l'ouverture du programme. Dans ce contexte, le dispositif installé par lartiste peut désormais osciller entre le régime de contraintes propre à la structure du programme et celui, plus aléatoire, dû à l'ouverture au-dehors qui caractérise certains projets dialogiques axés sur le contenu de la communication interactive. D'autres dispositifs visent en effet, au-delà de la généricité de l'algorithme, une possibilité de dialogue avec linteracteur: offrant au minimum un espace à parcourir de façon active et jusquà la possibilité d’un apport extérieur venant contaminer l'œuvre. 


\section{L'œurre interactive}

Les dispositifs interactifs composent la part la plus importante et la plus visible des réalisations avec et pour l'Internet. L'attention y est focalisée sur l'attribut d'interactivité que l'on peut décliner en quatre principaux soustypes :

- Les dispositifs à exploration mettent en scène une interactivité de navigation. Ils permettent de voir et d'expérimenter l'œuvre, qu'elle soit finie ou en cours de formation.

- Les dispositifs à contribution aménagent une interactivité de commande: l'exécution d'un algorithme de programmation partagé entre linteracteur et la machine. Ils permettent une activation de l'application plus qu’un réel apport de matériaux.

- Les dispositifs à altération concèdent aux interacteurs d'intervenir sur l'œuvre, dans le respect de règles et de procédures, par transformation ou apport de données. Ces dernières, matérielles ou immatérielles, visibles ou enfouies, auront une incidence sur le résultat final.

- Les dispositifs à alteraction initient un processus de communication permettant aux interacteurs de travailler collectivement, dans un jeu presque sans règles dont l'alteraction (c'est-à-dire la réaction en temps réel aux actions d'autres individus) est le fondement.

Le schéma suivant met en évidence le caractère hybride de ces différents types d’interactivité considérés. 


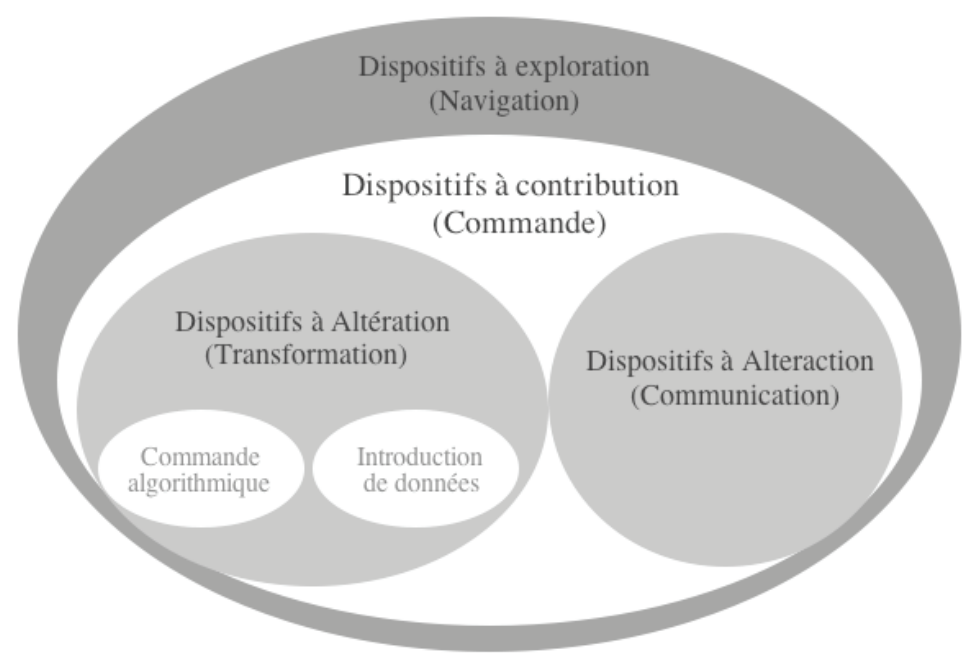

Figure 3. Types d'œuvres interactives

Il s'agit d'un système englobant -l'interactivité navigationnelle, dite à exploration, constituant la forme primaire de relation à l'œuvre du Net art à partir de laquelle sont développés des degrés d’interactivité techniquement plus élevés. La commande, l'altération ou l'alteraction composent les trois régimes d'interactivité des dispositifs à contribution. Pour chaque figure de l'interactivité, l'accent doit être mis sur l'apport de matériaux par le visiteur/acteur, la prise en compte et le rôle du temps dans le déroulement du processus, l'état de l'œuvre à un instant donné et sa transformation, la localisation des pièces du dispositif et la répartition de la puissance de calcul, les rapports interpersonnels dans l'observation, la conception ou la transformation de l'œuvre, sa localisation de l'œuvre, sa visibilité, sa durée.

Les dispositifs à exploration

La majeure partie des sites Web autorise au minimum l'exploration, mettant l'acteur en situation de naviguer. Ils permettent la découverte, plus ou moins ludique, de lieux préalablement disposés par l'artiste. Mais certains sites ne laissent aucune autre possibilité de transformation, l'interactivité se résumant à un certain ordre de déroulement, un parcours dans un stock immuable de formes. En ce sens, ils sont homogènes avec le mode courant de consultation sur le Web. Toutefois, l'accès à l’ouvre nécessite désormais un parcours actif 
de la part du visiteur. Cette ergodicité - pris au double sens de travail et de chemin - revêt des accents différents d'un dispositif à l'autre et engage des compétences spécifiques de navigation. En effet, un dispositif à navigation seule n'annonce pas inévitablement la simulation d'un parcours dans ce qui compose l'équivalent d'une galerie d'art où sont exposées des pièces et installations au regard de leur valeur strictement esthétique et plastique. Certains s'attachent au contraire à innover sur ce terrain de la lecture hypertextuelle en proposant des dispositifs inédits de narration et de scénarisation hypermédia. Les travaux du laboratoire "Esthétique de l'interactivité » ${ }^{26}$ offrent à cet égard des exemples très intéressants d'écriture multimédia centrée sur la notion de récit interactif.

Le mode réticulaire qualifie donc d'emblée l'ensemble des sites Internet, parmi lesquels certains dispositifs artistiques déclinent différentes acceptions plus élaborées du schéma d’arborescence. Centrés sur la visite, les sites Alteraction de Reynald Drouhin ou Lightmare de Michel Cleempoel permettent des actions ludiques et créatives sur différentes pièces interactives et immersives. Les pièces ainsi proposées à l'exploration demeurent toutefois des entités isolées et closes sur elles-mêmes, l'œuvre étant bien encore ici l'objet présenté27.

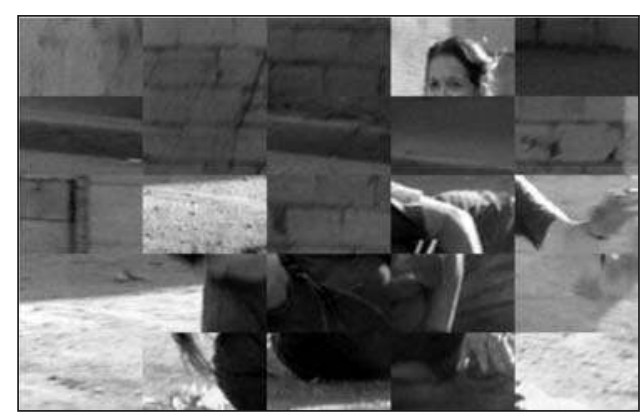

Figure 4. Samuel Bianchini, Sniper, www.dispotheque.org

\footnotetext{
${ }^{26}$ Sous la direction de Jean-Louis Boissier à l'Université Paris VIII.

${ }^{27}$ Cf. Drouhin, R., Alteraction: http://www.ensba.alteraction.fr et Lightmare de l'artiste Michael Cleempoel. Voir également les projets de Streaming vidéo ou autres animations générées par algorithmes d'anonymes.net, http://anonymes.net, de Flying Puppets, http://www.flyingpuppet.com, ainsi que les Boucles et collages de Shirin Kouladjie, http://www.n3xt.com.
} 
Le procédé à carte offre par exemple une mise en scène du site, au sens géographique du terme, qui permet de naviguer en ayant une représentation d'ensemble des différentes pièces ou lieux aménagés par l'artiste. Les dispositifs Virtual actions de Michael Blum, Be safe/be free de Valéry Grancher, S'élancer de La Cie, Taliesin de Didier Lechenne répondent à ce schéma ${ }^{28}$. L'autoportrait, extrait de Globus oculi29 (1992), procède également d'un système à carte innovant, utilisant la technologie Shockwave pour donner au visiteur l'illusion d'un contrôle continu sur une bille ${ }^{30}$. La navigation inclut ici une possibilité de jeu (variations) sur la forme (l'ordre) et son déroulement par laquelle linternaute peut modifier ce qu'il voit sans pour autant contrôler ce quil parcourt. Ce qui fait de linternaute un joueur et apparente son parcours à une expérimentation concrète.

La figure du labyrinthe, dans lequel le visiteur est invité à se perdre, constitue une troisième modalité possible de navigation -certains projets jouant de l'étendue du réseau et de ses infinis constituants interconnectés pour disposer des pièges et autres déviations venant perturber le parcours de linternaute. D’autres réalisations, telle La Maison des Immondes Pourceaux $^{31}$ de Nicolas Frespech, s'apparentent à des mises en scène de jeux d'aventure dans lesquels le visiteur doit lui-même engendrer son parcours. D'autres encore étendent le labyrinthe au-dehors de leur site même et vont jusqu'à délocaliser et cacher des fragments de leurs dispositifs en des espaces -derrière les pages- étrangers au projet artistique ${ }^{32}$.

Une dernière forme de cette navigation exploratoire résulte de l'organisation de Puzzle Ring, qui regroupe des sites dont chacun offre seulement une partie d'une image globale dont la recomposition et l'affichage nécessitent de parcourir la somme des espaces et sites où elle se déploie. Ce parcours hypertextuel et itinérant promeut une figure différente de la carte qui représente un territoire fragmenté. La navigation recourt en effet ici à la

\footnotetext{
${ }^{28}$ Cf. Grancher, V., be safe/be free, http://www.imaginet.fr/nomemory/data/safe.html. La CIE, S'élancer, http://www.icono.org/lacie/inven.htm. Lechenne, D., Talieslin, http://www.icono.org/lechenne/parcours.htm. Blum, M., Virtual actions, http://www.icono.org/blum/actions.htm.

${ }^{29} \mathrm{Cf}$. Boissier, J.-L., L'autoportrait, Globus Oculi, http://www.labart.univ-paris8.fr/ifi/jlb/02.htm.

${ }^{30}$ Le dispositif Flora Petrinsularis de JL Boissier offre également, sur cd-rom, une illustration du système à carte.

31 Cf. Frespech, N., Les immondes pourceaux, http://immondes.cicv.fr/pourceaux.html.

32 Voir par exemple le site de Easylife, http://www.easylife.org. Ou certains travaux de Jodi, http://www.Jodi.org.
} 
collecte d'informations et de matériaux dispersés en de multiples nœuds d'hébergement et de connexion. Divers projets déroulent de la sorte la métaphore du rhizome empruntée à Gille Deleuze (1980:32).

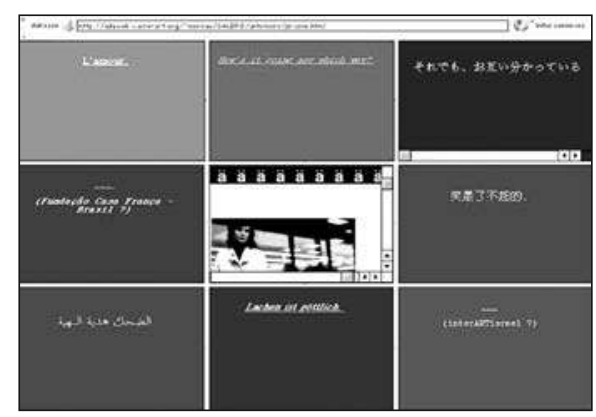

Figure 5. Antoine Moreau, On se comprend, http://antomoro.free.fr/comprend.html

C'est le cas notamment d'On se comprend développé par l'artiste Antoine Moreau ${ }^{33}$ - la partie immédiatement visible à l'écran n'étant dans ce projet qu'une portion d’un agencement de différents éléments en différents lieux. L'interface explorable, telle qu'elle apparait à l'écran, sous-tend en effet un dispositif plus complexe, composé de différents fragments informatiques dispersés sur le réseau dans 8 serveurs, ou sites miroirs, ainsi liés et reliés par l'artiste. On se comprend se présente sous la forme d'une page de 8 fenêtres, chacune d'entre elles contenant 12 phrases. Chaque phrase est traduite en 8 langues (anglais, chinois, arabe, hébreu, portugais, allemand, japonais et français) et correspond à un site hébergeant le projet. Il s'agit d'un système de multi-fenêtrage. Ce n'est qu'après avoir sollicité les I2 phrases d'une même fenêtre que le dispositif affiche dans la fenêtre centrale la page du site hébergeur de la portion d'œuvre correspondante. Ainsi, et même si l'artiste propose une explication sommaire et évoque des règles de jeu, ce n'est qu'à l'issue de ce parcours, que l'on peut entrevoir la nature du dispositif. Car, plus que d'afficher la page d'accueil du site hébergeur dans la fenêtre centrale, ce dispositif génère à notre insu notre déplacement dans les

33 Premier artiste accueilli en résidence de création d'une œuvre Web par le Centre International de Création Vidéo d'Hérimoncourt (CICV). Cf. Antoine Moreau, On se comprend, http://antomoro.free.fr/comprend.html. 
différents serveurs. Il n'y a ici rien de spectaculaire, le seul indice de ce déplacement est l'adresse http qui apparaît dans le menu Location de notre navigateur. On a effectivement basculé dans le fragment qui se trouve hébergé ici ou là. L'intention qui a présidé à ce travail était de produire une création qui soit simultanément présente en différents lieux, dans différents sites miroirs. Ainsi que le commente lui-même Antoine Moreau, "c'est ce qui m'intéressait, l'idée de manifester et d'amplifier des liens, l'ubiquité des dossiers qui se trouvent partout ».

Rhizomes $^{34}$, de l'artiste Reynald Drouhin, propose un dispositif voisin qui sollicite les machines-clients des internautes. L'objectif visé est ici l'hébergement de 192 fragments d'image sur autant de serveurs distants. L'ensemble constitué de ces fragments dispersés est destiné à être recomposé sur le serveur de Rhizomes pour ne former qu'une seule image. En ce sens, le dispositif re-présente limage ainsi constituée en même temps que la cartographie du réseau composée par l'ensemble des serveurs ainsi reliés 35 . Avec Nervures ${ }^{36}$, l'artiste Grégory Chatonsky retourne ce processus de délocalisation de l'œuvre, en se focalisant non plus sur les images mais sur l'are de convergence que leur mise en relation constitue. L'accent est ainsi mis sur lindexation physique et territoriale des fragments informatiques dispersés sur le réseau, ainsi que sur le caractère pleinement assignable de leurs parcours eux-mêmes. Cette entreprise de «traçage "37 permet en effet de rendre visible les chemins parcourus par les fichiers images d'un nœud à l'autre du réseau, d'une IP (Identification of Position) machine-client à une autre.

Ces différents dispositifs, axés principalement sur la navigation, composent encore la majeure partie des sites Web. Ils requièrent une lecture active et induisent des mécanismes de partage de la puissance de calcul très divers selon la généralité du processus envisagé. Pour lartiste, la navigation

\footnotetext{
${ }^{34}$ Cf. Drouhin, R., Rhizomes, http://zweg.cicv.fr/creation_artistique/online/rhizomes, 2001.

35 En outre, l'œuvre institue un contrat de maintenance entre l'artiste et l'internaute sollicité. Le mode d'emploi suppose en effet d'héberger une image et deux fichiers HTML envoyés par l'artiste, et de mettre a disposition son adresse (répertoire) à partir de laquelle pourra être reconstituée l'image réticulaire que les participants s'engagent à rendre visible à l'ensemble des internautes pour une durée déterminée. L'œuvre éphémère reposant sur cet engagement collectif des 192 internautes qui composent les différentes parties du rhizome.

${ }^{36}$ Cf. le dispositif de Chatonsky, G., Nervures, http://nervurat.io-n.net, le 08/02/2001.

${ }^{37} \mathrm{Cf}$. par exemple Trace Root qui permet de suivre au fil des liens le transport d'une information sur le réseau et/ou de dessiner des sessions de parcours des internautes en ligne.
} 
emprunte principalement le protocole Http, incluant sporadiquement le téléchargement d'applets Java sur la machine servie, la quasi-totalité de l'exécution des calculs intervenant postérieurement à ce téléchargement d'applets Java ou de modules de type Schockwave sur la machine-client. Pour linternaute, le Web est principalement sollicité comme transmetteur de l'information, l'essentiel des activités consistant en téléchargement et navigation. Ce qui se traduit ici par le déroulement d'un dispositif en temps réel, au sens du Web, et donc par le déploiement d’une œuvre qui se donne à voir et dont le scénario est généralement pré-écrit, y compris dans ses variations. Les chemins qui peuvent parcourir l'œuvre sont innombrables et le visiteur n'est précisément sollicité que pour naviguer et voir. L'auteur semble alors le créateur du site, ou parfois le metteur en scène de trajets qui en fait un scénariste au sens même des logiciels utilisés pour les mettre en forme.

\section{Les dispositifs à contribution}

Les dispositifs de cet ensemble comptent sur le visiteur en tant que contributeur à une création désormais désignée comme collective. Par conséquent, l'action de l'internaute s'exerce non seulement sur le parcours dans une ouvre dont tous les lieux ont été définis à l'avance, mais aussi sur sa création ou re-création. Ces dispositifs permettent en effet d'agir autant sur le parcours au sein du dispositif (exploration) que sur les formes et contenus qui le composent (contribution). La contribution, dont les répercussions ont une incidence directe sur l'ouvre, prend done une valeur performative. Suivant l'intrication des régimes d'interactivité présenté dans le diagramme précédent, il est possible de distinguer différents types de contribution: la génération d'une commande algorithmique, l'altération par apport de nouveaux matériaux et l'alteraction collective médiée par le dispositif. Je propose d'aborder successivement ces trois modalités de linteractivité qui engagent diversement les actions de l'internaute, l'exécution par la (les) machine(s) de scripts techniques et la localisation des constituants de l'œuvre.

La contribution la plus élémentaire - dite ici «de commande"- se limite à l'actualisation d’un processus algorithmique dont les règles et effets 
partiellement prédéfinis intègrent une dimension aléatoire. Telegarden ${ }^{38} \mathrm{de}$ l'artiste chercheur Ken Goldberg prolonge sur le réseau Internet les investigations de télé-présence. Le processus mis en œuvre pointe les incidences d'une interaction médiée, à mi-chemin entre le réel et le virtuel, partagée entre l'espace physique (Ars Electronica Center) et le cyberespace (via linterface Web du site). Il s'agit d'un jardin dont les internautes s'occupent à distance via un télé-robot. Ce dispositif déploie deux types d'interactions potentielles: un mode impliqué, vigilant et actif, visant l'entretien du jardin; et un mode non impliqué de visualisation d'une évolution et croissance des végétaux. Ce type de confrontation à distance promeut linstauration d'une communauté d'échange et exige une forme d'apprentissage de la part du public, qui ne va pas toujours de soi :

"Cette œuvre a suscité un immense scepticisme : parmi les gens qui voyaient le jardin dans son espace physique à l'Ars Electronica Center, certains n'arrivaient pas à croire que les plantes étaient arrosées, désherbées, plantées, etc., par une communauté d’internautes. Et parmi ceux qui se connectaient, certains n'arrivaient pas à croire qu'à des milliers de kilomètres de leur écran se trouvait effectivement un vrai jardin „39.

Par conséquent, la manipulation interroge le degré d’implication de linternaute et les incidences physiques de son action virtuelle. Dans ce contexte, le clic de souris a en effet pour résultat concret de générer l'intensité lumineuse nécessaire à la croissance d'une plante et donc de donner ou maintenir la vie. À linverse, linaction peut y être perçue comme une nonassistance susceptible d'engendrer la mort ${ }^{40}$. En interrogeant cette performativité de la télé-action, Ken Goldberg souligne l'illusion de réalité sous-tendue par ces dispositifs.

De nombreux autres dispositifs déploient des situations analogues d'hybridation de l'espace physique et du cyberespace. On trouve par exemple

\footnotetext{
38 Cf. Goldberg, K., Telegarden, http://telegarden.aec.at, créé à l'université de Californie du Sud en 1995 et présentée durant deux années consécutives à l'Ars Electronica Center de Linz (1996-97)

${ }^{39} \mathrm{Cf}$. Annick Bureaud, «Pour une typologie de la création sur Internet », sur le site de la revue Leonardo http://www.olats.org/OLATS/ivres/etudes/index.shtml, 1998.

${ }^{40}$ Sur cette problématique de l'implication, depuis l'espace potentiel d'Internet où naît l'action du visiteur à l'espace physique où elle aura des incidences sur l'installation de l'artiste, voir Goldberg, K., Shadow Server 1997 (littéralement, un «serveur d'ombres » couplé à un générateur automatique, en réponse aux requêtes des internautes) : http://taylor.ieor.berkeley.edu. Succédant au Telegarden, le projet Teleporting of an Unknown State d'Eduardo Kac (1996) propose également aux internautes de contribuer à la croissance d'une plante à distance. L'intensité lumineuse vitale est ici acheminée depuis les Webcams des internautes qui éclairent, au sens fort du terme, le processus de germination d'une graine : Home page : www.ekac.org/.
} 
dans cette catégorie les structures gonflables (Blimps)41 des artistes John Canny \& Eric Paulos, interfacées à l'Internet pour permettre une exploration spatiale. Ou encore, Light on the Net ${ }^{42}$ de l'artiste Masaki Fujihata qui rend modifiable par l'Internet une sculpture lumineuse disposée dans le hall d'un immeuble de bureaux à la Préfecture de Gifu au Japon. Plus métaphoriquement, Jarrête le temps de l'artiste Fred Forest offre aux internautes le pouvoir de contrôler le flux informationnel du réseau en gelant symboliquement une portion de temps. Cette Machine à travailler le temps $s^{43}$ déploie un double dispositif en et hors ligne : d'une part, sur le site Web est représentée une horloge que les internautes connectés peuvent choisir d'accélérer (bouton rouge) ou de ralentir (bouton vert); d'autre part, le programme informatique affiche le résultat de ces actions en avançant et reculant les aiguilles d’une deuxième horloge physiquement présente sur le site de l'exposition. À l'ubiquité spatiale promue par la télé-présence, ce dispositif superpose l'étirement ou la condensation du temps, médiés et pris en charge par laction quiil délègue au public. Il confronte de la sorte les différentes inscriptions temporelles des participants - en et hors ligne- à la vitesse de transmission des informations par l'Internet.

Pour l'ensemble de ces dispositifs, l'interactivité à commande contributive associe un système d'affichage sur la machine-client à une procédure de récolte de l'information vers la machine-hôte. Ces dispositifs instaurent de la sorte un double processus, décomposable en procédures d'envoi et de réception, qui tire parti des informations générées par l'action des internautes et à partir desquelles l'œuvre est modifiée puis réaffichée dans son nouvel état. L'action de linternaute ne se limite donc plus ici à une consultation -même active- des lieux disposés par l'artiste, elle intègre désormais la commande d’un algorithme de programmation.

Les dispositifs à altération

Ces dispositifs permettent, de surcroit, l'intégration de données apportées par les internautes au cours de leur expérimentation. Cette nouvelle implication peut revêtir des formes différentes: la participation à un vote, l'envoi d'un texte, d'une image ou d'un son. Ici, la programmation

41 Cf. Telembodiement (1997). http://www.prop.org.

42 Cf. Fujihata, M., Light on the Net, http://www.flab.mag.keio.ac.jp/Light.html.

43 Cf. Forest, Fred., Machine à travailler le temps, http://www.fredforest.org/temps, Fête de l'Internet (1998). 
algorithmique permet simultanément la captation de ces données et la gestation d'une nouvelle figure: une métamorphose de l'œuvre par apports de matériaux externes. L'interactivité spécifique de ces dispositifs permet en effet aux internautes de participer en commun et en temps différé à la transformation ou à l'évolution d'une création artistique. L'œuvre résultant de ces systèmes incrémentaux est cumulative, elle " grossit " avec le temps et au fil des contributions. De nombreux dispositifs s'apparentent ainsi à des archives ou bases de données d’informations.

Depuis I992, l'artiste et universitaire américaine Bonnie Mitchell ${ }^{44}$ développe des projets collaboratifs reliant de cent à trois cents personnes dispersées dans une vingtaine de pays différents ${ }^{45}$. ChainReaction initié en I995 à l'occasion du Siggraph et de l'ISEA ${ }^{46}$ à Montréal, compose une chaîne artistique collaborative qui s'étend continuellement à mesure que des individus participent autour du monde. Le dispositif invite linternaute à choisir une image, sur un catalogue de trente-deux images initiales, dans l'objectif de la modifier. Cette image lui est prêtée pendant trois heures. Après quoi, l'image modifiée doit être renvoyée au site qui l'ajoute à sa base d'images déjà constituée et à son catalogue. Cette nouvelle image pourra à son tour être choisie par un participant et ainsi de suite. Chaque image travaillée donne en effet naissance à une lignée dans laquelle une image mère ne peut avoir que deux enfants. La structure proposée est done celle d'un arbre binaire qui se développe au gré des participants. Une particularité intéressante de ChainReaction est l'automate qui se cache derrière: les actions de l'utilisateur sont surveillées par un programme sur le serveur, qui gère les prêts d'images et l'arrivée des nouveaux matériaux. Ce programme, écrit en langage Perl, a un nom évocateur; il s'agit d'un moteur de collaboration dont le processus est le suivant: le participant soumet une demande sur formulaire électronique, réservant une image pour trois heures ; les données du formulaire sont transférées dans une base de données; la personne manipule (modifie) l'image chez elle, en temps différé, avec le logiciel de son choix, et renvoie le résultat de son travail au site; le

\footnotetext{
${ }^{44}$ Bonnie Mitchell est artiste et professeur à la Syracuse University, College of Visual and Performing Arts, School of Art and Design, Department of Art Media Studies.

45 Cf. Mitchell, B., ChainArt en 1992, Digital Journey en 1993, Diversive Paths en 1994 et Chain Reaction, initialement développé sur http://chain.syr.edu/index.html et aujourd'hui sur http://creativity.bgsu.edu/collaboration/ChainReaction/index.html (1995).

${ }^{46}$ L'International Symposium of Electronic Arts (ISEA).
} 
programme regarde si l'image attendue en retour est revenue ; lorsqu'elle est revenue, le programme place l'image dans son dossier et en fait trois versions à des tailles différentes, une page HTML est générée pour supporter limage grandeur nature, et l'image aux formats plus petits se propage dans les autres pages du site pour devenir des boutons d'accès. Le moteur recueille aussi le nom de chaque participant et alors que la personne qui va prendre une image est nommée participant, une fois limage renvoyée on la désigne comme artist et on l'autorise de ce fait à signer son œuvre. Le nombre d'images constituées à partir des premières était de l'ordre de $35^{\circ}$ au bout d'environ quatre ans de fonctionnement, le but assigné étant de I oo० images.

D’un principe assez semblable au dispositif précédent, le protocole d’usage de As Worlds Collide ${ }^{47}$, très écrit, suppose d’altérer un espace de réalité virtuelle. Le projet collaboratif intègre les principes des environnements en trois dimensions (Time-based expressive worlds), il compte I77 participants. À l'instar d'une image fixe, il est ainsi désormais possible de manipuler un environnement virtuel navigable. Les internautes sont dans ce cas sollicités comme co-créateurs de mondes virtuels donnés à voir et à explorer.

D’autres dispositifs, moins ambitieux, concèdent également au public la possibilité de nourrir une base de donnée évolutive. Dans Topoi ${ }^{48}$, Valéry Grancher invite par exemple les internautes à décrire leur lieu de vie par email. L'ensemble des textes reçus est ensuite affiché dans linstallation enligne qui grossit en taille et finit par constituer une architecture domestique archétypale. Les pièces Alone ${ }^{49}$ et Identities déploient un dispositif similaire de recueil par e-mail de témoignages de personnes contaminées par des épidémies. Ce dispositif qui joue de l'anonymat et de la confidentialité de ces communications sur le réseau, rejoint ensuite une installation dans l'espace

${ }^{47}$ Cf. Bonnie Mitchell, As Worlds Collide, http://creativity.bgsu.edu/collaboration/worlds/index.html, 1997/1998. Cette production distribuée mobilise différentes ressources informatiques. Pour créer les images de départ (starter world), les artistes emploient des techniques de photographie numérique, la 3D et les logiciels de retouche -les images ainsi formées étant ensuite converties en animations QTVR Panorama et dirigées par un script d'Apple. Afin de les transformer-, l'utilisateur doit lui-même disposer du plugin QTVR, des diverses versions d'applications sollicitées par l'édition des images ainsi que du logiciel NetPresenz (ftp) qui permet de transférer les images vers le As Worlds Collide. Enfin, l'ensemble de ce processus -la surveillance du site, la récupération des images et leur conversion (du format Pict au Jpg) - est autogéré par un script en langage Perl.

${ }^{48}$ Cf. Grancher, V., Topoï,

http://www.imaginet.fr/nomemory/data/place.htm (1998).

${ }^{49}$ Cf. Grancher, V., Alone, http://www.nomemory.org/data/alone.htm (1999). 
physique, au musée CAPC de Bordeaux, composée de plaques de cire scellant à tout jamais l'ensemble de ces confessions anonymes. Les travaux plus récents de l'artiste utilisent les techniques innovantes de composition d'images-mosaïques à partir de la mise en réseau des Webcams d'internautes, dans Be safe/be free ${ }^{50}$, Webscape ${ }^{51}$ ou encore les moteurs de recherche pour composer des portraits d'internautes, dans Search Art ${ }^{2}$. L'œuvre Des Frags de lartiste Reynald Drouhin constitue un aboutissement de cette forme d'altération d’images prélevées sur le réseau, réemployées pour composer avec la complicité des internautes de nouvelles images mosaïques.

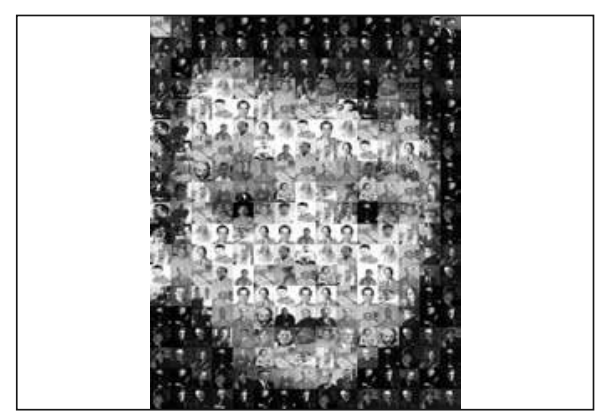

Figure 6. Reynald Drouhin, Des Frags, www.desfrags.cicv.fr

D’un principe analogue, Autour de l'écran ${ }^{53}$, développé en 1997 par l'artiste Simon Tric, établit une association informatiquement médiée entre des écrivains et des dessinateurs. Les premiers sont appelés à décrire leurs bureaux aux seconds qui, grâce à leur interprétation des textes recueillis, en proposent une illustration graphique. Par linjonction «dessine-moi mon bureau ", se superposent à l'écran d'ordinateur les descriptions brouillées d’images physiques et métaphoriques de différents bureaux, où naissent le

\footnotetext{
${ }^{50}$ Cf. Grancher, V., Be safe/be free, http://www.nomemory.org/data/safe1.html (1999).

${ }^{51}$ Cf. Grancher, V., Webscape, http://www.nomemory.org/data/mind.html (2000) composée d'une mosaique faite de 100 animations numériques animées selon des combinaisons aléatoires liées à plus de 50 caméras temps réel réparties dans tous les points du monde.

52 Cf. Grancher, V., Search Art, http://www.nomemory.org/search, 2001, qui utilise les tags et codes du moteur de recherche le plus conventionnel - google - comme outils pour élaborer des pièces portraits «search art ».

53 Cf. Simon Tric, Autour de l'écran, http://www.icono.org/tric/propos.htm, 1997.
} 
travail et limaginaire artistique contemporains. Dans ce dispositif, linternaute choisit un rôle (écrivain ou dessinateur); il est payé de son effort par un accès aux différents autres textes et dessins. À l'inverse, le nonparticipant ne peut entrevoir qu'une fraction du site, la plus grande partie étant réservée aux acteurs directement impliqués ${ }^{54}$.

Au cœur de ces projets, laction interactive est ainsi cadrée par de strictes prescriptions d'emploi. Les matériaux doivent être envoyés conformément aux modalités générales énoncées par le projet, qui énonce des règles explicites de collaboration et informe les internautes des opérations attendues ainsi que de leurs incidences sur le dispositif. On part done d'une sorte d'état initial de l'œuvre, puis le travail se fait à plusieurs, croît en importance avec le temps et avec le nombre de participants dans un schéma d'évolution prédéfini. Le rapport aux autres contributeurs se fait dans une relation I-n : un contributeur face aux autres qui ont déjà travaillé sur l'œuvre. Le temps différé permet à l'interacteur d'organiser son travail à sa guise, la seule contrainte étant d'expédier un document au format souhaité par le site collecteur. L'œuvre, quant à elle, se voit et se manipule, mais le dispositif mis en place pour permettre son existence et son développement peut lui aussi être considéré comme une œuvre.

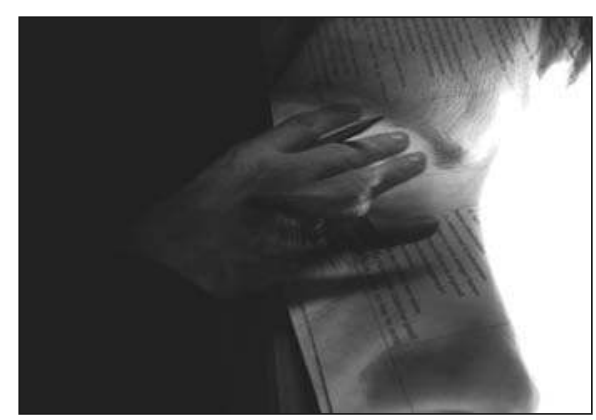

Figure 7. Grégory Chatonsky, Sur Terre, www.arte.tv/sur-terre

\footnotetext{
${ }^{54}$ La vue de l'œuvre nécessite ici encore une forme active d'engagement de la part de l'internaute. Le dispositif imposant de manière stricte ses conditions d'emploi, cadrées par l'existence d'un contrat liant l'artiste et son public.
} 


\section{Les dispositifs à alteraction}

Cette dernière sous-catégorie de dispositifs à contribution permet aux internautes de collaborer non plus en différé mais en temps réel à l'émergence d'une ouvre collective. Autrement dit, l'alteraction, envisagée ici comme une action intermédiaire qui fait devenir autre (Quéau I988), engage de multiples participants dispersés dans la conception simultanée d'entités discrètes, par la mise en relation desquelles émerge une ouvre autonome globale.

Un des rares exemples satisfaisant à ce modèle d’interactivité est le Générateur Poïétique ${ }^{55}$ développé par l'artiste-ingénieur Olivier Auber. Ce dispositif déploie une expérience d'interaction graphique collective acentrée, qui forme le support de recherches sur les phénomènes collectifs temps réel ${ }^{56}$. Le principe du Générateur poïétique permet en effet à plusieurs individus de se connecter, à un moment donné, sur un site -le lieu et l'heure du rendez-vous ayant été fixés par courrier électronique - afin de participer à la création d'une image commune. La mise en œuvre du dispositif implique le téléchargement d’un logiciel assez rudimentaire de dessin bitmap dont l'apprentissage est immédiat. La modification de limage se fait en continu et en temps réel. Chacun pour soi modifie limage que tous voient, altérant formes et couleurs.

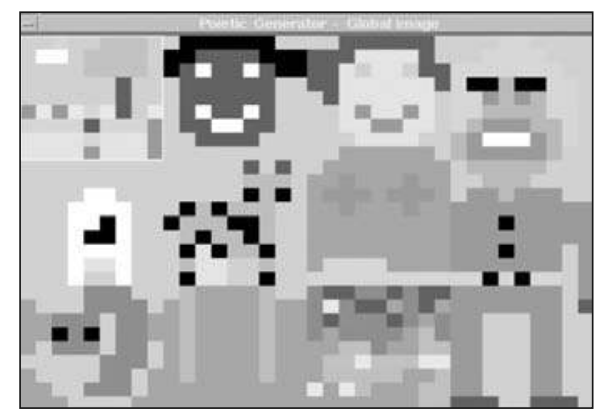

Figure 8. Olivier Auber, Le Générateur poḯtique, http://poietic-generator.net

\footnotetext{
55 Cf. Olivier Auber, Le Générateur poḯtique, http://poietic-generator.net, 1986/2004.

56 «Un système autopoïtique est organisé comme un réseau de processus de production de composants qui régénèrent continuellement par leurs transformations et leurs interactions le réseau qui les a produits, et qui constituent le système en tant qu'unité concrète dans l'espace où il existe, en spécifiant le domaine topologique où il se réalise comme réseau ». Francisco Varela (CNRS/École Polytechnique).
} 
L'action de chacun, qui est donc visible par l'ensemble des participants, apparait déterminée par l'état de l'image à un instant donné, elle-même résultat de laction de tous. Par conséquent, au coeur de l'expérience du Générateur Pö̈́tique, la communication semble importer davantage que ce qui est produit, limage qui en résulte n'ayant pas plus d'intérêt que n'importe quelle image intermédiaire. Initialement lancé en 1986 sur des plateformes Minitels ${ }^{57}$, ce projet a été expérimenté lors de différentes manifestations artistiques aussi bien que scientifiques. À partir de 1995, une version pour le Mbone (le backbone Multicast de l'Internet dédié à la recherche) est développée par des étudiants et chercheurs de l'École Nationale Supérieure des Télécommunications (ENST, Paris). Linfrastructure du réseau y est utilisée avec le protocole particulier Multicast qui permet une interaction "tous-tous» en temps réel, sans recourir à un serveur central ${ }^{58}$. Autrement dit, là où le dispositif à altération permettait de constituer petit à petit un objet de manière incrémentielle et en temps différé, le mode alteractif permet de vivre et de sentir en temps réel l'action et la réaction simultanées entretenues avec les autres participants. Envisagé dans cette perspective, le premier propose un dispositif de transformation de l'œuvre, alors que le second promeut davantage un dispositif d'échanges entre individus. L'orientation de recherche déployée par ce projet créatif engage donc un "processus cognitif » distribué, axé sur l'émergence de formes et de normes d'un travail artistique en réseau régi par des mécanismes de contagion et d'hybridation, des rythmes inhérents à chaque individu, couplés à linstrument technique ${ }^{59}$.

\section{Une esthétique de l'interactivité}

Il ressort de cette analyse différentes modalités de la relation interactive avec ou à travers les dispositifs Net art. Ces relations engagent tout autant la notion d'œuvre, sa localisation et sa requalification, que les modalités de sa conception et réception active. Le travail artistique en réseau renouvelant en

\footnotetext{
${ }^{57}$ Cf. Principalement, Communication et monumentalité, Centre Georges Pompidou, Paris, 1990, et Machines à communiquer, Cité des Sciences et de l'Industrie, Paris, 1992.

58 Instaurant une modalité de connexion inédite, différente de la relation «un-tous » spécifique des systèmes broadcast comme la radio ou « un-un » propre au système unicast comme le téléphone.

${ }^{59}$ Au fil de ce processus, l'artiste identifie des cycles de vie propres, des moments où l'on voit émerger des « supers signes qui succèdent à une première phase chaotique, puis de nouveau le chaos et ainsi de suite ».
} 
effet significativement les modalités du partage de savoir et de compétences dans l'activité entre les différents participants aux prises avec des dispositifs techniques. À lisssue de cette analyse focalisée sur la déclinaison des figures de linteractivité, nous pouvons mieux appréhender ces régimes d'action et d'attribution mis en ouvre par les dispositifs du Net $\operatorname{art}^{60}$.

Chacune de ces figures, reprises dans le tableau ci-dessous, prévoit ainsi des emplois et des incertitudes, des contraintes et des prises par lesquelles se co-construisent l'action et l'objet, ses schémas de circulation et ses régimes d'existence. L'interactivité minimale y est toujours navigation dans un espace d'information plus ou moins transparent et arborescent. Une interactivité plus complexe peut prescrire la génération d’un algorithme de programmation. Dans ce cas, elle est simultanément commande d'un processus observable pour l'acteur du dispositif et branchement algorithmique pour l'auteur. Une troisième relation interactive peut encore consister en la possible introduction de données de la part de l'acteur. Il s'agit là d'une interactivité de contribution, cette dernière pouvant ou non avoir une incidence réelle sur le contenu ou la forme de l'œuvre. Dans ce cas, la contribution est doublée d’une altération. Enfin, linteractivité peut être le terreau d’une communication inter-humaine médiée, et c'est alors l'alteraction -l'action collective en temps réel- qui compose le cœur du projet artistique.

\section{Partition synthétique des dispositifs interactifs}

La notion de dispositif permet d'entrevoir la façon dont le site a été disposé par l'artiste. Le dispositif regroupe et configure différents niveaux de l'œuvre. Le dispositif anticipe le rôle et la part attendue du visiteur dans le processus de création.

\footnotetext{
${ }^{60}$ Notons que ces figures de l'interactivité, ici distinguées pour des raisons analytiques, co-existent parfois en un même dispositif, mais au sein duquel elles n'ont toutefois pas le même poids.
} 


\begin{tabular}{|c|c|c|}
\hline Types de dispositifs & Interactivité/interaction & Temporalité \\
\hline $\begin{array}{l}\text { Dispositifs à exploration } \\
\text { Antoine Moreau, On se } \\
\text { comprend } \\
\text { http://antomoro.free.fr/comprend. } \\
\text { html }\end{array}$ & $\begin{array}{l}\text { - Navigation seule } \\
\text { - Parcours de lien en lien } \\
\text { - Dispositifs prédéfinis } \\
\text { - Pas de transformation } \\
\text { - Interactivité machine }\end{array}$ & $\begin{array}{l}\text { - Temps de la navigation } \\
\text { - Temps du spectateur } \\
\text { - Réhabilitation du trajet } \\
\text { - Rapport sélectif-actif } \\
\text { - Parcours linéaire }\end{array}$ \\
\hline $\begin{array}{l}\text { Dispositifs à } \\
\text { contribution } \\
\text { M.Benayoun, Et moi dans tout } \\
\text { Ça } \\
\text { http://www.benayoun.com/indexF } \\
\text {.html }\end{array}$ & $\begin{array}{l}\text { - Visiteur contributeur } \\
\text { - Apport et transformations } \\
\text { - Dispositifs participatifs }\end{array}$ & $\begin{array}{l}\text { - Temps de l'œuvre } \\
\text { - Temps de l'artiste }\end{array}$ \\
\hline $\begin{array}{l}\text { Dispositifs à altération } \\
\text { Bonnie Mitchell, Chain } \\
\text { Reaction } \\
\text { http://creativity.bgsu.edu/collabor } \\
\text { ation/ChainReaction/index.html }\end{array}$ & $\begin{array}{l}\text { - Participation en commun } \\
\text { - Transformation } \\
\text { - Actions isolées } \\
\text { - Alternance } \\
\text { - Inscriptions différées } \\
\text { - Relation asynchrones }\end{array}$ & $\begin{array}{l}\text { - Temps différé } \\
\text { - Temps de l'œuvre } \\
\text { - Systèmes incrémentaux }\end{array}$ \\
\hline $\begin{array}{l}\text { Dispositifs à alteraction } \\
\text { O. Auber, Le Générateur } \\
\text { poḯtique } \\
\text { http://perso.enst/ auber }\end{array}$ & $\begin{array}{l}\text { - Participation conjointe } \\
\text { - Collaborations } \\
\text { - Mode alteractif } \\
\text { - Synchronicité } \\
\text { - Réciprocité } \\
\text { - Simultanéité }\end{array}$ & $\begin{array}{l}\text { - Temps réel } \\
\text { - Temps de la collaboration } \\
\text { - Temps de l'émergence }\end{array}$ \\
\hline
\end{tabular}

Cette mise en relation à actants répartis permet ainsi de qualifier des régimes d'existence et d'actions à l'œuvre dans le Net art. Chaque type d'interactivité engendre une économie symétrique de dispositions et d'usages. Par exemple, les dispositifs privilégiant la qualité formelle ou informationnelle visent davantage une interactivité d'exploration dans une base de données finalisée. Au mieux, ils offrent une possibilité de commande et d'exécution du déroulement d'une animation. La modalité relationnelle correspondante est généralement de type intra-actionniste, s'apparentant en cela à un art du cliquable et nimpliquant que la seule relation homme/machine. À l’opposé, le dispositif communicationnel est focalisé sur linterface de la mise en relation et offre des prises inter-actionnistes aux participants. L'analyse de ces frontières de la désignation et délimitation de 
l'œuvre dépend en grande partie de ces modes hétérogènes de relation et d'usages rapportés ici aux dispositifs.

L'analyse proposée permet de distinguer trois principales formes du Net art : les ouvres de contamination médiatique, les œuvres de génération algorithmique et les œuvres de communication interactive. Les premières sont principalement axées sur l'interface (médiologique) par laquelle transitent l'œuvre, l'usage et la communication. Les deuxièmes sont focalisées sur le programme (algorithmique) d'objets-animations ou d'objetsenvironnements qui laissent ou non à linternaute la possibilité d’interagir. Les troisièmes sont focalisées sur le contenu interactif de ce qui est disposé, variant de l'objet arborescent (engageant un parcours réticulaire), à l'objet en devenir (concédant un parcours altérant) et jusqu’à l'objet-relation (distribuant un parcours inter-communicationnel). De ce point de vue, l'œuvre médiatique a pour objet le matériel digital, l'œuvre algorithmique a pour objet le programme et, enfin, l'œuvre interactive prend pour objet la communication (formelle) et ce qui en résulte.

Ces différents types mettent par conséquent clairement en évidence ce glissement par lequel l'œuvre se trouve moins dans ce qui est donné à voir que dans le dispositif qui la fait exister. L'affichage sur l'écran n'étant le plus souvent que la face apparente de toute une infrastructure technique et informationnelle, l'œuvre devient alors, de façon plus large, l'ensemble des structures et des règles qui la sous-tendent. Ce caractère fragmenté de l’œuvre en réseau rend heuristique l'approche focalisée sur le dispositif, si l'on accepte de concéder à ce concept plus de flexibilité et d'ouverture en l'écartant de sa seule indexation au champ de linstrumentalité. En effet, lusage du concept de dispositif marque ici un changement par rapport à la régulation technique classique en introduisant le recours à de nouveaux moyens d'action sur l'œuvre, tels que, notamment, la délégation et la décentralisation. Le dispositif n’y est donc plus un intermédiaire qui vient se situer entre les sujets communicants, mais un environnement dans lequel ces derniers entrent activement (Duguet I998, 2002). Autrement dit, la mise en œuvre du dispositif, initiée par l'artiste, se déploie en diverses opérations de montage socio-technique et d'organisation de séquences confiées au visiteur. 
C'est là en effet toute l'ambivalence du dispositif, placé entre une configuration technique et un cadrage social de laction. Celui-ci peut être entendu simultanément en tant que machine et en tant que mécanisme, dans la mesure où il sous-tend à la fois l'acte et la manifestation artistique en aménageant différentes prises (Bessy \& Chateauraynaud 1994) en direction d'un public qui peut désormais, selon certaines réserves et conditions, devenir l'acteur de fragments de l'œuvre laissés ouverts. D'autres travaux ont pointé les mutations récentes du comportement des publics: amateurs (Hennion, Maisonneuve \& Gomart 2001) ou consommateurs (Cochoy 2004) ou même innovateurs (Caelen 2004) auxquels on reconnaît aujourd'hui d'importantes compétences, sociales et techniques (Dodier 1993), qui plaçent ainsi l'usage au cœur de l'innovation, de la création ou de la recherche participatives. Le Net art met ainsi en scène un dialogue médié par lordinateur qui opère un déplacement et un décentrement progressif des instances traditionnellement impliquées dans le processus de communication. Focalisée sur la relation auteur/acteur, l'analyse de ces nouveaux dispositifs demande en effet que soit réévalué le modèle communicationnel distribué entre l'émission, le message et la réception -la médiation de lordinateur contrariant ici la représentation d’un message isolable, strict médiateur entre un auteur et un lecteur. Le curseur glisse désormais entre ces trois domaines d'énonciation, pour simultanément souligner leur autonomie et l'hybridation réciproque de leurs composantes, là où l'œuvre matérialise un "champ de possibles ». Entre l'activité rationnelle, instrumentale, et la passivité contemplative et réceptrice d'un environnement, l'entre-deux du dispositif pointe plutôt vers l'idée de médiation. D’une part, il met en jeu des matériaux à demi particularisés et adaptables aux circonstances. D'autre part, dans ce contexte, l'exhiber n'est pas rationnellement structuré et tendu vers une fin en soi (la conclusion ou la solution) - le dispositif contribuant au contraire, par la mise à disposition des éléments qui le composent, à susciter le travail cognitif du visiteur. Par conséquent, l'analyse de ces objets ne pourra désormais plus être focalisée sur leur attribut ontologique ou sur la croyance qui les maintient et les entretient, mais devra être élaborée au fil des prises successives quils engagent et par lesquelles ils se déploient : des objets repris, transformés, et transformateurs, sur le mode impur d'une action mêlée, située, collective, passant par de multiples dispositifs et inscrite dans une double histoire. L'histoire de l'art 
contemporain, qui depuis les années 1960 se caractérise par une volonté de rompre les cloisons entre l'artiste et le public. L'histoire des médias, en quête de nouvelle formes d'attachement et de relations mieux distribuées entre dispositifs techniques et pratiques sociales.

J.-P. F.

\section{Références}

Akrich, Madeleine

1990 "De la sociologie des techniques à une sociologie des usages".

Techniques et Culture I6 : 83-IIO.

1993 «Les objets techniques et leurs utilisateurs. De la conception à laction », in Conein, Dodier \& Thévenot (eds), « Les objets dans laction. De la maison au laboratoire ", Raisons Pratiques 4 : 35-58.

Arasse, Daniel

1999 Le Détail. Pour une histoire rapprochée de la peinture. Paris : Flammarion.

Barthes, Roland

1975 "En sortant du cinéma », in Le Bruissement de la langue : Essais critiques IV. Paris : Éditions du Seuil : 407-412.

Bessy, Christian \& Francis Chateauraynaud

1995 Experts et faussaires. Pour une sociologie de la perception.Paris : Métailié.

Bouriaud, Nicolas

I998 L'Esthétique relationnelle. Paris : Les Presses du réel.

Caelen Jean (ed.) 2004 Le Consommateur au cour de linnovation. Paris : CNRS Éditions.

Cochoy, Franck (ed.)

2004 La Captation des publics. C'est pour mieux te séduire, mon client... Toulouse : Presses Universitaires du Mirail. 
De Certeau, Michel

I990 L'Invention du quotidien, Tome. I : « Arts de faire ». Paris :

Gallimard («Folio/Essais »).

Deleuze, Georges

I980 Mille Plateaux. Paris : Éditions de minuit.

Duguet, Anne-Marie

I988 "Dispositifs ", in Communications. Paris : Seuil : 22I-242.

2002 Déjouer limage. Nimes : Jacqueline Chambon.

Dodier, Nicolas

1993 "Les arènes des habiletés techniques ", dans Conein, Dodier et Thévenot, Les objets dans laction. De la maison au laboratoire. Raisons Pratiques, $n^{\circ} 4$, Paris, 1993, EHESS, p. II5-139.

Eco, Umberto

I965 L'Euvre ouverte. Paris : Le Seuil.

Fourmentraux, Jean-Paul

2004 "Quête du public et tactiques de fidélisation. Une sociologie du travail et de l'usage artistique des NTIC ", Réseaux I24 : 8I-II2.

2005 Art et Internet. Les nouvelles figures de la création, Paris : CNRS Éditions.

Gell, Alfred

1998 Art and Agency. An Anthropological Theory. Oxford: Clarendon Press

Genette, Gérard

I996 L'Euvre de l'art. Tome 2, La relation esthétique. Paris : Seuil.

Goodman, Nelson

1996 L'Art en théorie et en action. Paris : Éditions de l'Éclat.

Hennion, Antoine

I993 La Passion musicale. Une sociologie de la médiation. Paris : Métailié.

Hennion, Antoine, Sophie Maisonneuve \& Emilie Gomart

2000 Figures de l'amateur. Formes, objets, pratiques de l'amour de la

musique aujourd'hui. Paris : La Documentation Française.

Latour, Bruno

I994 "Une sociologie sans objet ? Remarques sur l'interobjectivité ".

Sociologie du travail $4: 587-608$.

1999 « Factures / Fractures. From the concept of network to the concept of attachement ". RES, $36: 20-3 \mathrm{I}$

Marin, Louis

I994 De la représentation. Paris : Le Seuil. 
Norman, Donald

I993 "Les artefacts cognitifs ", in B. Conein, N. Dodier \& L. Thévenot, Les Objets dans laction. De la maison au laboratoire. Paris : Éditions de l'EHESS (Raisons Pratiques, ${ }^{\circ} 4$ ) : I5-34.

Strauss, Anselm

1992 La Trame de la négociation. Paris : Éditions L'Harmattan.

Thévenot, Laurent

1990 "Laction qui convient ", in P. Pharo, L. Quéré, Les Formes de l'action. Paris : Éditions de EHESS : 39-69.

1994 "Objets en société, ou suivre les choses dans tous leurs états ». Alliage 20 et $21: 74-88$.

Woolgar, Steve

I99I "Configuring the user : the case of usability trials. ", in John Law, $A$ Sociology of Monsters : Essays on Power, Technology and

Domination, London : Routledge : 57-99. 